\title{
Surgical Management of Herniated Intrathoracic Gastric Perforation in Traumatic Diaphragmatic Rupture: An Unusual Two Rare Cases
}

\author{
Isaac Okyere ${ }^{1,2 *}$, Sanjeev Singh ${ }^{2,3}$, Perditer Okyere ${ }^{2,4}$, Samuel Gyasi Brenu', \\ Samuel Kontor Amoabeng 5 \\ ${ }^{1}$ Department of Surgery, Komfo Anokye Teaching Hospital, Kumasi, Ghana \\ ${ }^{2}$ School of Medicine and Dentistry, College of Health Sciences, Kwame Nkrumah University of Science and Technology and \\ Komfo Anokye Teaching Hospital, Kumasi, Ghana \\ ${ }^{3}$ Department of Anaesthesia and Intensive Care, Komfo Anokye Teaching Hospital, Kumasi, Ghana \\ ${ }^{4}$ Department of Medicine, Komfo Anokye Teaching Hospital, Kumasi, Ghana \\ ${ }^{5}$ Department of Pharmacy, Komfo Anokye Teaching Hospital, Kumasi, Ghana \\ Email: ${ }^{*}$ rokyere@yahoo.com
}

How to cite this paper: Okyere, I., Singh, S., Okyere, P., Brenu, S.G. and Amoabeng, S.K. (2020) Surgical Management of Herniated Intrathoracic Gastric Perforation in Traumatic Diaphragmatic Rupture: An Unusual Two Rare Cases. Open Journal of Thoracic Surgery, 10, 56-68.

https://doi.org/10.4236/ojts.2020.102006

Received: May 28, 2020

Accepted: June 27, 2020

Published: June 30, 2020

Copyright $\odot 2020$ by author(s) and Scientific Research Publishing Inc. This work is licensed under the Creative Commons Attribution International License (CC BY 4.0).

http://creativecommons.org/licenses/by/4.0/

\begin{abstract}
Gastric perforation into the thoracic cavity through a diaphragmatic rupture is rare but, when it occurs, patients present in severe distress, with mortality approaching $50 \%$. We present our experience of two rare and unusual cases of traumatic diaphragmatic rupture from penetrating chest injury associated with herniated intrathoracic gastric perforation over a five-year period from January 2015 to December 2020 at the cardiovascular and thoracic surgery department of the Komfo Anokye Teaching Hospital in Kumasi, Ghana. Both patients underwent successful surgical repair through standard posterolateral thoracotomy with one having earlier negative exploratory laparotomy. The essence of the paper is to share and discuss the clinical presentation, diagnostic challenges, surgical management and the postoperative care of this very rare complication of traumatic diaphragmatic rupture.
\end{abstract}

\section{Keywords}

Diaphragmatic Rupture, Gastric Perforation, Penetrating Chest Injury, Thoracotomy

\section{Introduction}

Traumatic diaphragmatic rupture, either due to blunt or penetrating thoracic or 
abdominal injuries, is a rare occurrence with reported incidence of less than $10 \%$. Traumatic diaphragmatic rupture is usually associated with intrathoracic herniation of intraabdominal organs and intestines. However, the occurrence of traumatic diaphragmatic rupture with herniated gastric perforation is a rare presentation with few reported cases in literature [1]. The diagnosis is often difficult and a delay in diagnosis frequently results in increased morbidity and mortality. The purpose of this paper is to share our experience in the management of these cases, to sensitize the readers about this rare entity and also discuss the diagnostic dilemma along with potential challenges in its management.

\section{Case Reports}

\subsection{Case 1}

The first case was a 46-year old male who was allegedly attacked by assailants who stabbed him twice in the left posterior chest. He started bleeding profusely from the wound sites with associated left-sided pleuritic chest pain and abdominal pain. He was rushed to the accident and emergency centre with the impaled knife in-situ at the back. He was however fully conscious but tachycardic with pulse rate of $111 \mathrm{bpm}$, moderate volume, BP of $144 / 93 \mathrm{mmHg}$, respiratory rate of 22 cycles per minute with saturation of $96 \%$ in room air. Air entry was reduced at the left middle and lower zones with stony dull percussion note and vesicular breath sounds. He had a $5 \mathrm{~cm}$ laceration over the left suprascapular area which admitted the whole length of the middle finger as well as a $4 \mathrm{~cm} \mathrm{la-}$ ceration $2 \mathrm{~cm}$ below the inferior angle of the left scapula with the dagger in situ. Immediate resuscitation with IV fluids, antibiotics, and antitetanus prophylaxis was started and a size $28 \mathrm{Fr}$ left chest tube was inserted draining bubbles of air and $300 \mathrm{ml}$ of non-clotting haemorrhagic effusion. He was thus consented for exploratory laparotomy and was wheeled immediately to the operating theatre after blood has been taken for grouping and matching against 4 units of blood, full blood count and chemistry.

Laboratory investigations done preoperatively showed haemoglobin level of $12.9 \mathrm{~g} / \mathrm{dl}(11.0-18.0)$, white cell count of $19.18 \times 10^{3} / \mathrm{uL}(3.00-15.00)$ with predominant neutrophilia of $80.9 \%$ (37.0 - 72.0) and Platelet count of $150 \times 10^{3} / \mathrm{uL}$ (150 - 400). Renal function was normal. Chest X-ray done showed a left haemopneumothorax as shown in Figure 1.

Using an extended upper midline laparotomy, the abdomen was entered. Findings were an intact left diaphragmatic contour, traumatic ileal perforation of about $3 \mathrm{~mm}$ in diameter and $40 \mathrm{~cm}$ from the ileocecal junction. All other intra-abdominal organs were normal. The ileal perforation was repaired and abdomen closed up in layers.

Postoperatively, patient started experiencing respiratory distress with respiratory rates of 40 cycles per minute, $\mathrm{SPO}_{2}$ of $94 \%$ on 6 litres of intranasal oxygen, pulse rate of $120 \mathrm{bpm}$ and temperatures above $38.4^{\circ} \mathrm{C}$ from postoperative day 3 after starting sips of liquids. Air entry was significantly reduced on the left mid- 


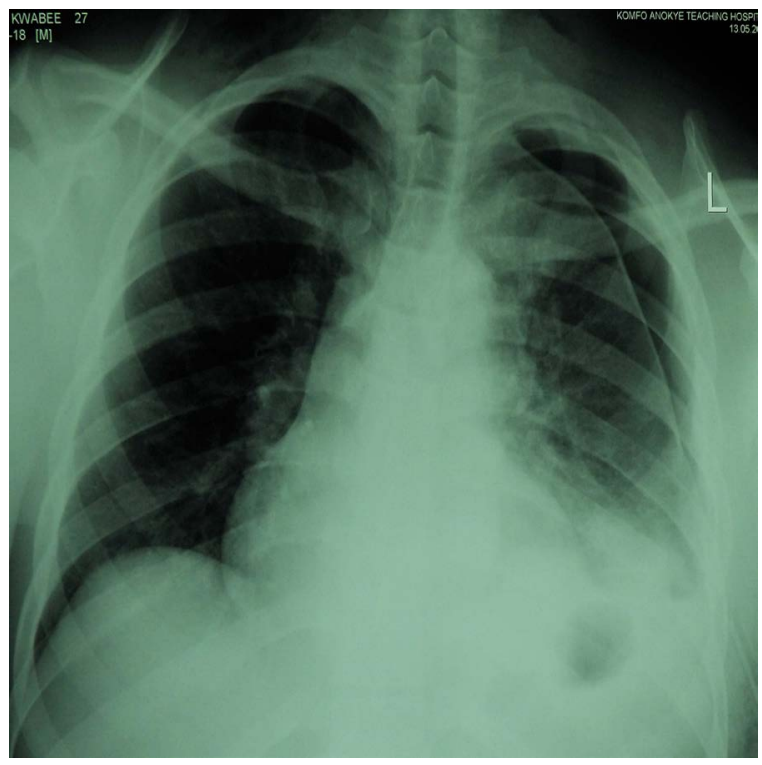

Figure 1. Chest X-ray showing Left Haemopneumothorax.

dle and lower zones with vesicular breath sounds. The chest tube which had been removed on postoperative day 2 was quickly repassed draining $800 \mathrm{mls}$ of brownish fluid of juice drunk earlier on and gush of air and a size 16Fr Nasogastric tube passed. The respiratory distress improved but temperatures were persistently above $38^{\circ} \mathrm{C}$ with chest tube now draining frank pus by postoperative day 4. Repeat complete blood count showed WBC of $22.83 \times 10^{3} / \mathrm{uL}$ with significant neutrophilia of $75.3 \%$, haemoglobin concentration of $9.7 \mathrm{~g} / \mathrm{dL}$ and platelet count of $412 \times 10^{3} / \mathrm{uL}$.

An urgent water-soluble upper GI contrast study on postoperative day 5 due to the suspicion of a possible intrathoracic oesophageal perforation showed evidence of reflux of gastric content with leakage noted at the distal oesophagus into the left pleural space as shown in Figure 2. After finally seeking an informed consent following patient's request to be discharged against medical advice and adequate optimization considering the subacute clinical course, an exploratory left thoracotomy was done on postoperative day 7. Entering the chest through a standard left posterolateral thoracotomy incision entering the $7^{\text {th }}$ intercostal space. Intraoperative findings as shown by Figure 3 and Figure 4 included:

- $2.0 \mathrm{~L}$ of frank purulent fluid.

- Frozen left hemithorax.

- Dense Fibrinous adhesions between the lobes of the lungs, the lungs and the chest wall covering and sealing the perforated stomach and the left hemidiaphragm.

- A $3 \mathrm{~cm}$ lacerated intrathoracic partially herniated and obstructed stomach which has been sealed by the fibrinous exudates.

- $4 \mathrm{~cm}$ diameter laceration of the left hemidiaphragm.

Careful blunt and sharp adhesiolysis was done to free the lobes of the left lung, the lung from the chest wall and the perforated stomach from the lower lobe of 

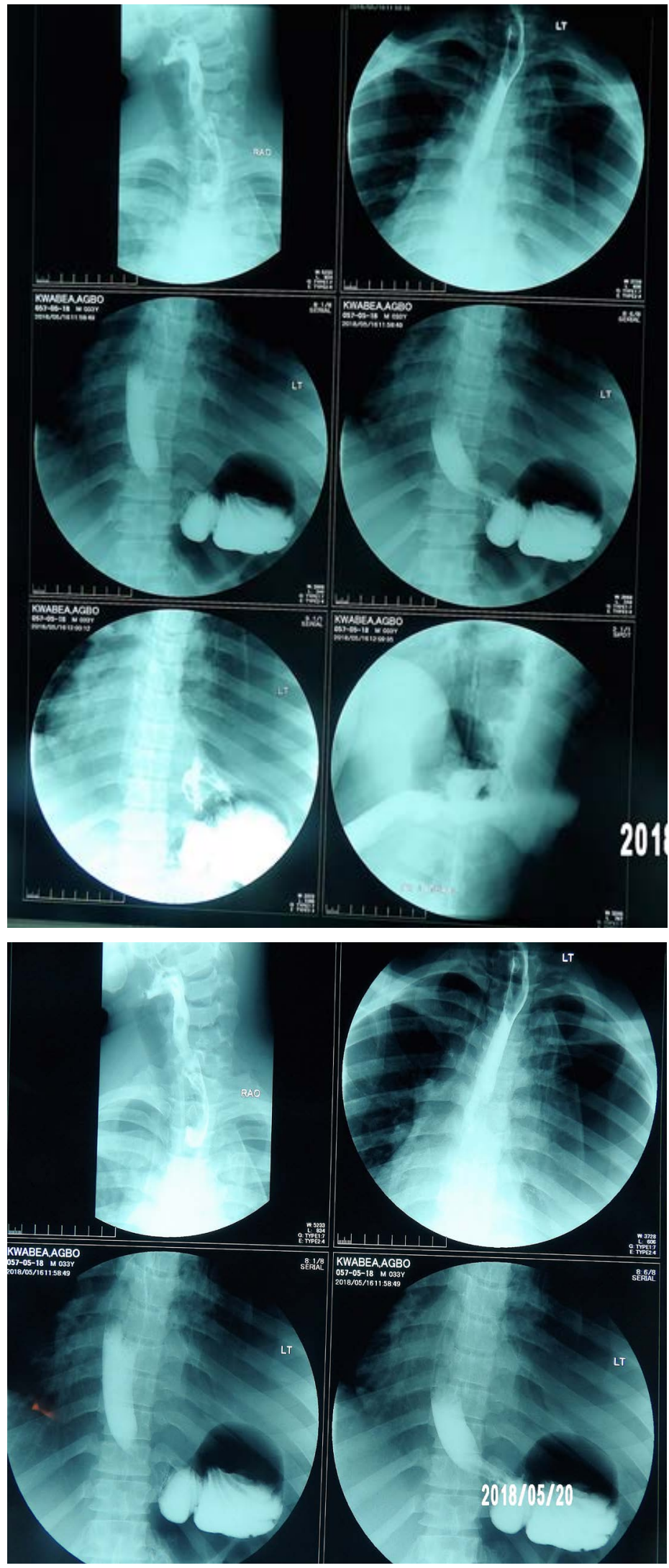

Figure 2. Water-soluble contrast study showing contrast spilling into left hemithorax. 


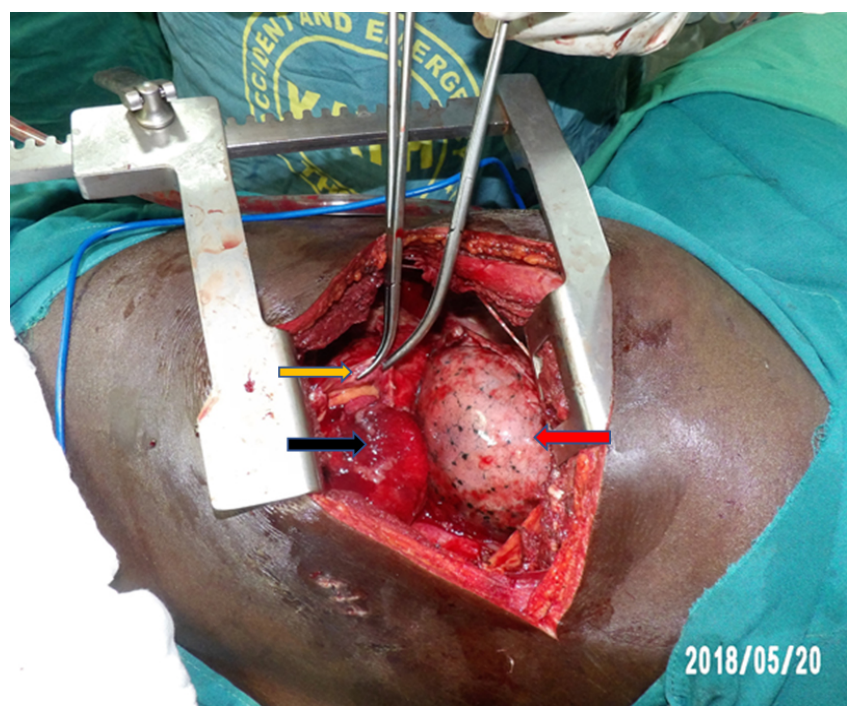

Figure 3. Showing the repaired Intrathoracic stomach with edges of the ruptured diaphragm; Black arrow: herniated intrathoracic stomach: Orange arrow: edges of ruptured diaphragm: Red arrow: lower lobe of left lung.

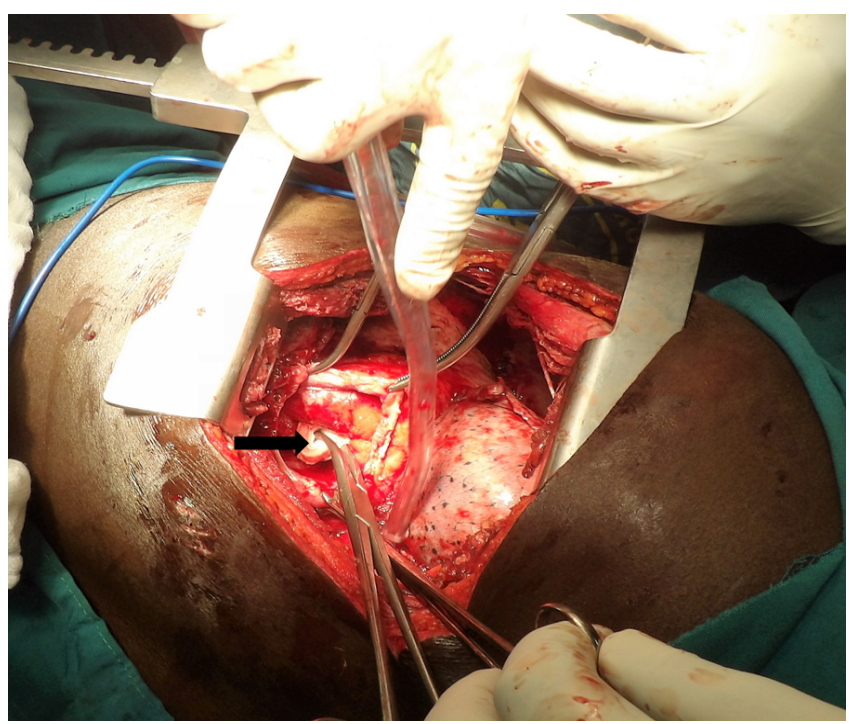

Figure 4. Black arrow: showing the edges of the diaphragm after reduction of the stomach into the abdomen.

the left lung and the diaphragmatic surface as well. The stomach was decompressed using the NG Tube and also directly through the perforation. The ischaemic edges of the stomach as shown in Figure 3 and Figure 4 were then freshened and the perforation was repaired in 2 layers with vicryl 3/0 in an interrupted fashion. It was then reduced into the abdomen after enlarging the diaphragmatic laceration. The diaphragmatic laceration was repaired with nylon 1 in a single-layered interrupted suturing as shown in Figure 5. The thoracic cavity was copiously irrigated with 1.0 litre of $0.9 \%$ Normal Saline and the thoracotomy incision closed up in layers over a size 32Fr new chest tube. 


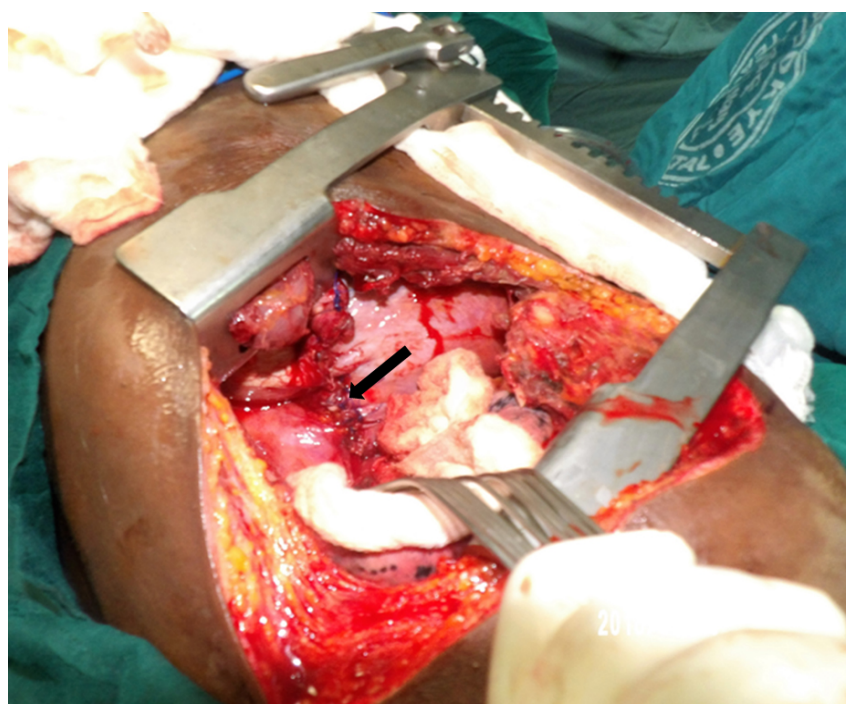

Figure 5. Black arrow: Showing the repaired diaphragm.

Patient made an uneventful recovery and was subsequently discharged home on postoperative day 7 . He was followed up on out-patient basis at 2 weeks, 1 month and 3 months and has been well since. Later he reported of his hypertrophic scars and was subsequently referred to the plastic surgeon for management.

\subsection{Case 2}

A 30-year-old male presented to the accident and emergency centre of our hospital with complaints of left-sided pleuritic chest pain associated with dyspnoea following a knife stab injury to his left lateral chest which he sustained three hours prior to presentation following a fight. On presentation he looked stable haemodynamically with a blood pressure of $120 / 88 \mathrm{mmHg}$, pulse rate of 126 bpm, good volume, respiratory rate of 26 cycles per minute and saturating at $94 \%$ on room air. Air entry was reduced on the left hemithorax with vesicular breath sounds but no added sounds. He had a $5 \mathrm{~cm} \times 2 \mathrm{~cm}$ deep laceration at the $8^{\text {th }}$ intercostal space between the midaxillary and posterior axillary lines. EFAST showed decreased lung sliding with extension of vertebral line on left hemithorax suggestive of left haemopneumothorax but negative haemopericardium and haemoperitoneum.

He had left chest tube insertion with size $32 \mathrm{Fr}$ tube draining bubbles of air and $550 \mathrm{mls}$ of blood. He was given antitetanus prophylaxis and blood was taken for grouping and cross-matching against 4 units of blood, full count and chemistry. Urgent chest X-ray as shown in Figure 6 showed an elevated left hemidiaphragm with gastric bubble in left hemithorax. However, after one hour the chest tube had drained about 1.7 litres of blood and therefore the patient was consented for and wheeled to the operating theatre for exploratory thoracotomy.

All laboratory investigations were in normal parameters before theatre. Under aseptic conditions and general anaesthesia with cuffed endotracheal intubation, 


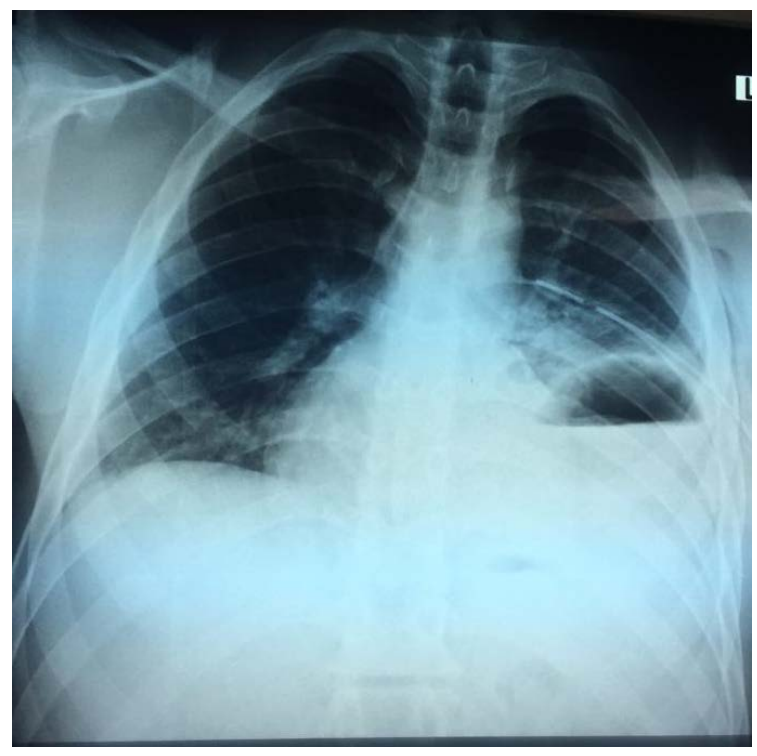

Figure 6. Preoperative chest X-ray showing elevated left hemidiaphragm and chest tube in situ.

a standard left posterolateral thoracotomy was done entering the $7^{\text {th }}$ intercostal space.

Intraoperative findings as shown in Figures 7-9 included, $1.5 \mathrm{~L}$ of haemorrhagic effusion, a $1 \mathrm{~cm}$ gastric perforation at the body of the herniated stomach with intrathoracic leakage of gastric contents, herniated greater omentum and a $10 \mathrm{~cm}$ transverse laceration of the left hemidiaphragm. The edges of the gastric perforation were freshened and it was then repaired in an interrupted doublelayered fashion with vicryl 3/0. The repaired herniated stomach, greater omentum were then reduced into the abdomen. The diaphragm as shown in Figure 10 was repaired in single layer by simple interrupted fashion with nylon 1 . The thoracic cavity was copiously irrigated with $0.9 \%$ Normal Saline and closed up in layers over a size 32Fr new chest tube.

Patient was extubated on table but spent three days in the intensive care unit and was subsequently stepped down to the ward. The chest tube was removed on postoperative day 3. He remained clinically stable and subsequently discharged home on postoperative day 7. He has since been well, being reviewed on out-patient basis at two weeks, one month and three months carrying his normal activities. The fully expanded lungs from his chest X-ray shown in Figure 11 and the nicely healed thoracotomy scar four months after the surgery as observed at the clinic.

\section{Literature Review}

\subsection{Background}

Traumatic diaphragmatic rupture from either penetrating or blunt thoracic or abdominal injuries is a rare occurrence with incidence of less than 10\%. Majority of the cases are from high velocity thoracic or abdominal injuries while $25 \%$ of 


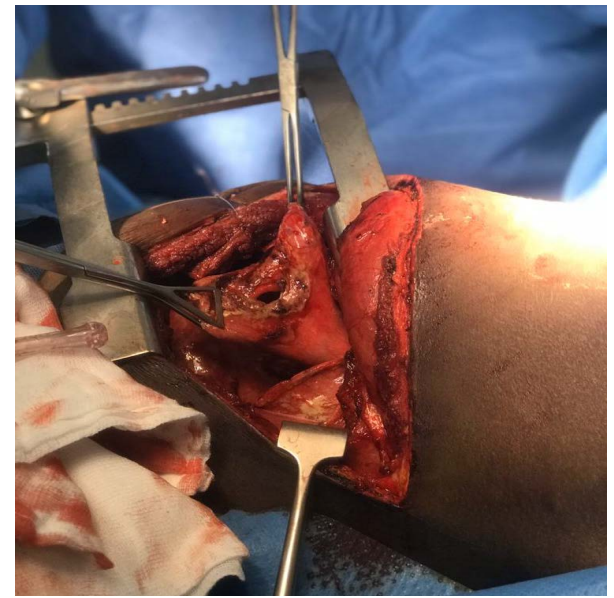

Figure 7. Showing the thoracotomy with the ischemic edges of the gastric perforation.

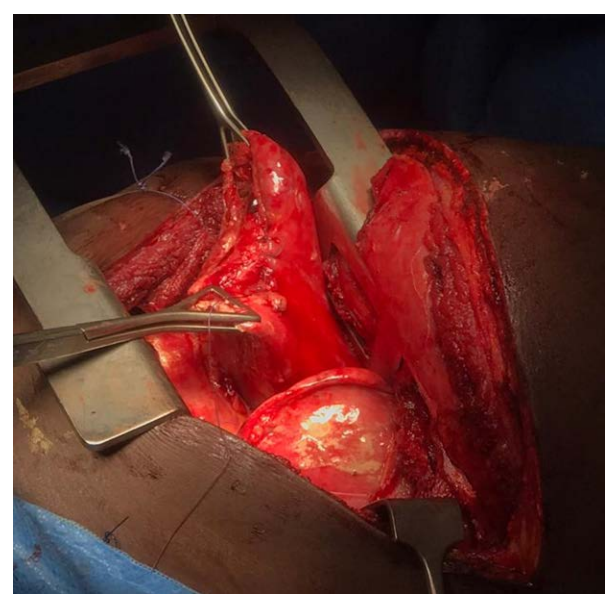

Figure 8. Intraoperative photo showing the repaired gastric perforation.

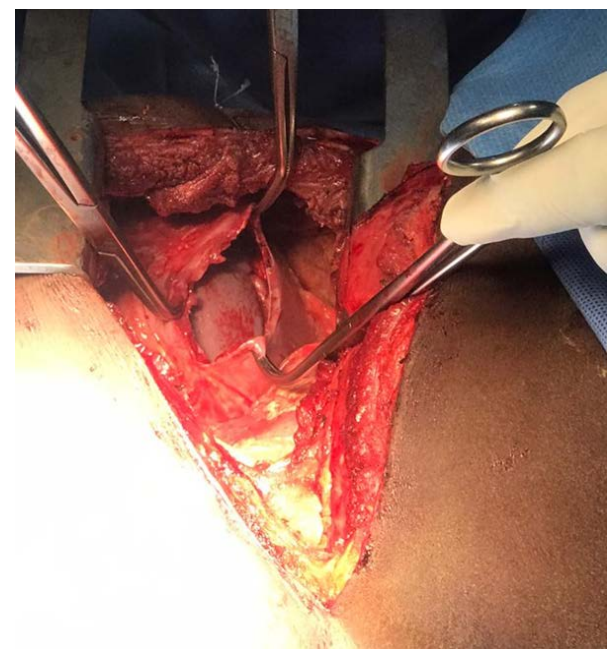

Figure 9. Showing the edges of the ruptured diaphragm after reduction of the repaired herniated intrathoracic stomach and the greater omentum. 


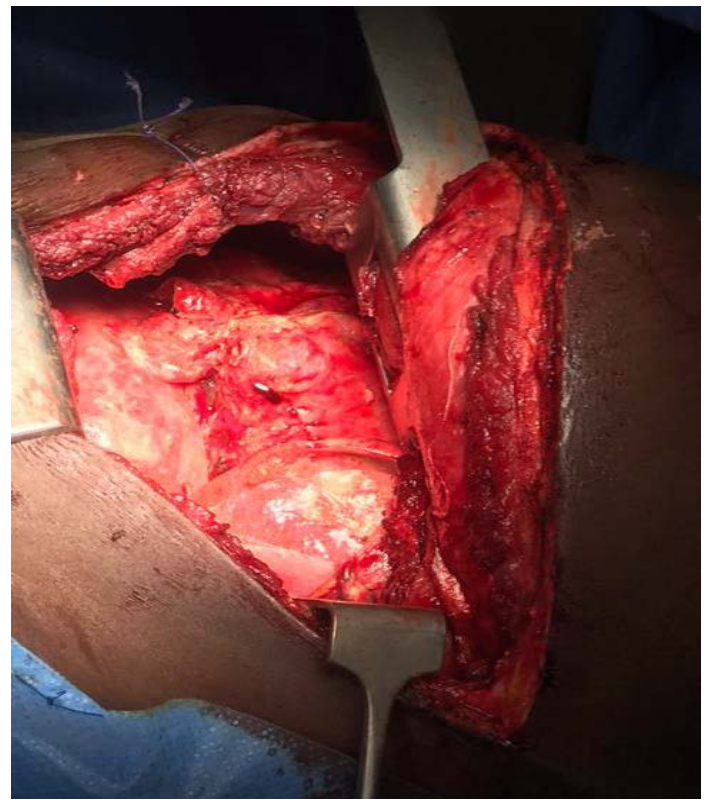

Figure 10. Intraoperative photo showing the repaired diaphragm.
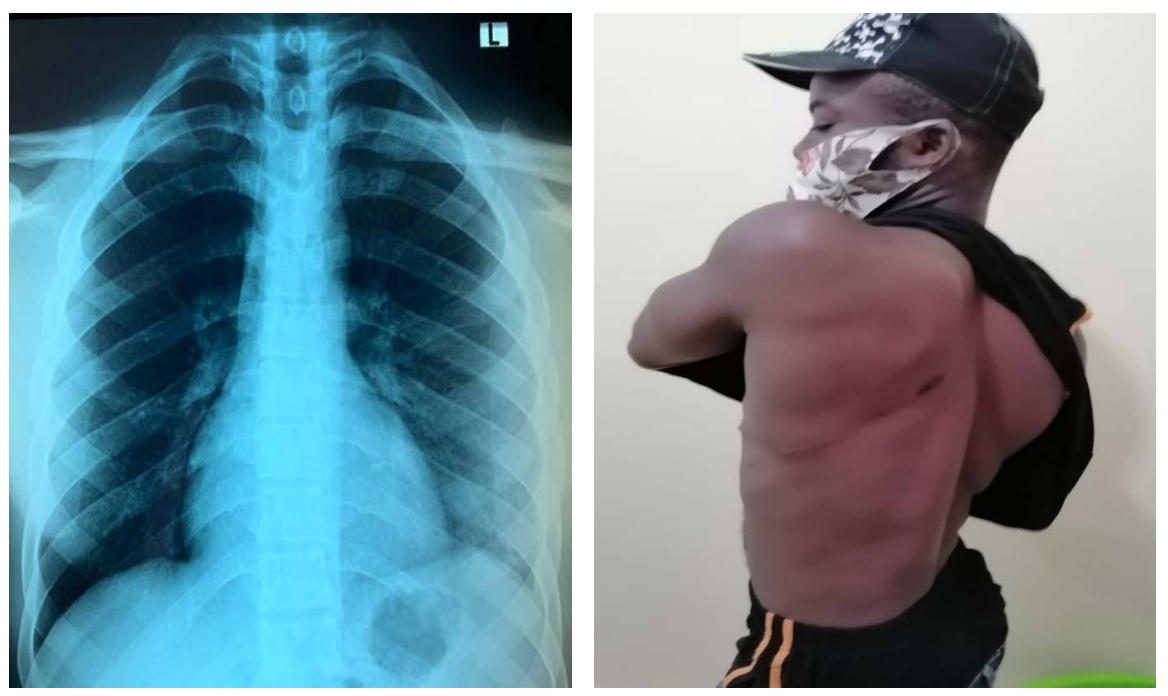

Figure 11. Showing repeat chest X-ray and the patient 4 months after surgery.

the cases arise from penetrating injuries such as from gunshot or stab injuries [2] [3]. The two cases presented in this paper resulted from penetrating chest injuries from knife stab wounds. Rare cases of spontaneous diaphragmatic rupture have been reported in literature from causes such as coughing, vomiting, sneezing, vaginal delivery or exercise [2] [4]. This type usually occurs on the left hemidiaphragm with up to $80 \%$ prevalence. The right-sided diaphragmatic ruptures occur in about $25 \%$ of the cases. The predominance of left-sided diaphragmatic ruptures have been attributed to the protective effect of the liver on the right, congenital weakness of the left hemidiaphragm and the relative underdiagnosis of right-sided diaphragmatic rupture due to the high mortality asso- 
ciated with the kind of high energy impact required to cause it [2] [5]. Penetrating trauma tend to cause smaller diaphragmatic defects than those seen in blunt trauma-related injuries. Therefore, they are more prone to complications such as bowel obstruction and strangulation [6]. The diaphragmatic rupture of our first case was missed during the initial exploratory laparotomy due to the small laceration from the knife. The subsequent leakage from the gastric perforation and mediastinitis led to the definite diagnosis leading to further investigation and the patient finally undergoing thoracotomy to repair both the stomach and the diaphragm and also to clean the thoracic cavity.

Traumatic Diaphragmatic injury with intrathoracic herniation of intraabdominal organs is a rare but potentially fatal condition particularly if accompanied by strangulation or intrathoracic perforation and spillage [1] [2] [4]. Traumatic diaphragmatic rupture with herniation may occur after thoracic or thoracoabdominal trauma either through blunt or penetrating trauma. It may go unnoticed initially only to present later on as a complication such as incarceration, intestinal obstruction or perforation [2] as it was in our first case.

One of the most serious complications of traumatic diaphragmatic herniation is intrathoracic perforation of a herniated hollow viscus, particularly the stomach. Such a double blow injury is particularly life-threatening since it contributes to development of mediastinitis, empyema thoracis or severe intra-parenchymal infections which are associated with significant morbidity and mortality [6]. Our first patient developed all these complications and had delayed diagnosis. Very few cases of traumatic diaphragmatic herniation with gastric perforation following blunt thoracoabdominal injuries have been reported in literature. Paswan and colleagues reported of an 18-year-old male who presented with a similar case of traumatic diaphragmatic rupture which was later complicated by gastric herniation and perforation 6 weeks after the trauma [1]. These injuries tend to arise due to a sudden increase in intra-abdominal or intrathoracic pressure following blunt trauma against a fixed diaphragm. It may also be due to shearing forces acting on a stretched diaphragm or diaphragmatic avulsion from its attachment [1] [7].

\subsection{Clinical Presentation}

Traumatic diaphragmatic injuries tend to have an insidious course and as such require a high index of suspicion to be diagnosed clinically [3]. Most commonly presented symptoms associated with traumatic diaphragmatic injuries include abdominal pain, dyspnoea, epigastric pain, shoulder pain and vomiting [3] [4]. Accurate preoperative diagnosis of traumatic diaphragmatic herniation has been possible in less than $50 \%$ of cases. Clinical presentation has been described in three phases, that is acute, latent or delayed and obstructive phases. The acute phase is predominantly associated with immediately life-threatening conditions such as haemorrhagic shock, respiratory failure, severe head injury or significant visceral injury. Hence, diagnosis of concomitant diaphragmatic rupture and/or 
herniation is frequently missed especially with a chest X-ray since they are usually inconclusive [1] [2]. The delayed phase usually presents with upper gastrointestinal symptoms, dyspnoea and chest pain or asymptomatic with abnormal chest X-ray [2]. The obstructive phase, a rare but potentially life-threatening event, usually is associated with incarceration and obstruction of herniated viscera [1] [4]. Clinical suspicion of traumatic diaphragmatic rupture should be raised in the event of a thoracic injury with concurrent abdominal symptoms. The reverse also holds true for abdominal injuries with concurrent thoracic symptoms [2] [3].

Traumatic diaphragmatic rupture with intrathoracic gastric perforation was found in $19 \%$ of the 22 cases with diaphragmatic rupture analysed by Akar and Kaya in 2017 [8]. Mortality associated with traumatic diaphragmatic rupture with intra-thoracic gastric perforation has been quoted by Vinnicombe et al. (2016) to be as high as $46 \%-52 \%$. Clinically, traumatic diaphragmatic injuries should be suspected in any penetrating injury from the level of the nipple to the umbilicus laterally and below the inferior pole of the scapula posteriorly [5].

\subsection{Diagnosis}

Chest X-ray is the initial screening imaging of choice for the detection of traumatic diaphragmatic ruptures even though diagnostic accuracy is about $30 \%$ in left-sided injuries and $18 \%$ in right-sided injuries [1] [2]. Yilmaz et al. (2006) showed that chest X-rays could be diagnostic in $25 \%-50 \%$ of cases. Diagnostic accuracy is reduced in the event of an accompanying pleural space collection and in patients with injuries due to penetrating trauma [2] [6] [9]. The pathognomonic signs on chest X-ray for the diagnosis of diaphragmatic rupture include the presence of gastric bubble, bowel loops in the hemithorax and intrathoracic presence of nasogastric tube. Other suggestive features include irregular diaphragmatic contour and elevated hemidiaphragm [2] [6] [7] [10]. CT scan of the chest is the diagnostic modality of choice with a higher sensitivity and specificity in diagnosing traumatic diaphragmatic ruptures [1] [5]. Other imaging modalities such as magnetic resonance imaging, ultrasonography and gastrointestinal contrast studies have all been employed in diagnosis [6] [9]. Diagnostic laparoscopy or thoracoscopy may be employed in cases with inconclusive findings following use of other radiological modalities [8].

Traumatic diaphragmatic herniation of the stomach or bowels can be easily mistaken for a pneumothorax or hydropneumothorax in the acute phase or it may be obscured by the presence of pleural space pathologies such as pneumothorax, haemothorax or haemopneumothorax or empyema thoracis. Such conditions mandate an emergency passage of an intercostal drainage tube, a procedure which can cause perforation of any undiagnosed herniated viscera. This scenario was classically reported by Yilmaz and friends in a case report where a 35-year-old male was diagnosed with a left haemopneumothorax following a penetrating injury to the left posterior chest. Patient started draining previously 
eaten food following chest tube passage [6]. Despite having chest tubes passed for the two patients before definite diagnosis and surgery, it is difficult to ascribe the perforation of the stomach to the chest tube since they were all penetrating knife stab wounds and the chest tubes were not found in the stomach when the chest was entered via thoracotomy.

\subsection{Management}

Traumatic diaphragmatic herniation is diagnosed incidentally at surgery or autopsy in $41.3 \%$ of cases while delayed diagnosis is seen in about $15 \%$ of cases [2]. The options for surgical repair include open exploration and repair by thoracotomy, laparotomy or thoracoabdominal incisions, or minimally-invasive methods such as laparoscopy and thoracoscopy [1] [2] [4] [6]. Following adequate patient resuscitation in acute cases, laparotomy allows rapid exploration of both hemidiaphragms and repair of the rupture as well as opportunity to thoroughly examine and repair any associated intra-abdominal organs particularly when there are no significant intra-thoracic injuries. Many authors also prefer to use thoracotomy approach even in acute cases especially when there are no associated intra-abdominal injuries and thoracotomy is also preferred in right-sided ruptures [2] [4] [6]. Okyere et al. from Ghana reported of two rare cases of right-sided diaphragmatic rupture with hepatothorax which were both repaired successfully via thoracotomy [5]. Thoracotomy is also particularly invaluable in patients with delayed presentation of diaphragmatic herniation so as to allow adequate adhesiolysis and easy repair of the rupture [2].

Traumatic diaphragmatic herniation with perforated hollow viscus contributes significantly to intra-abdominal abscesses, empyema thoracis or lobar pneumonia. As such, copious irrigation of the cavity with sufficient drainage is mandatory to limit risk of development [6] as was done for the two cases.

\section{Conclusion}

The association of traumatic diaphragmatic rupture with herniated intrathoracic gastric perforation is extremely rare with an associated high morbidity and mortality. The diagnosis needs high index of suspicion. Rapid resuscitation and early surgery via thoracotomy save lifes even in less resource centres.

\section{Acknowledgements}

Grateful to the patients from whom informed consent was obtained for the publication of the cases and accompanying images.

\section{Conflicts of Interest}

The authors declare no conflicts of interest regarding the publication of this paper.

\section{References}

[1] Paswan, S.S., Kumar, A., Chitta, M.R., Sudipta, M. and Saurabh, S. (2020) Double 
Blow Injury: Diaphragmatic Rupture with Gastric Perforation Following Blunt Trauma. SN Comprehensive Clinical Medicine, 2, 128-131. https://doi.org/10.1007/s42399-019-00167-1

[2] Dwari, A.K., Mandal, A., Das, S.K. and Sarkar, S. (2013) Delayed Presentation of Traumatic Diaphragmatic Rupture with Herniation of the Left Kidney and Bowel Loops. Case Reports in Pulmonology, 2013, 1-4. https://doi.org/10.1155/2013/814632

[3] Kara, E., Kaya, Y., Zeybek, R., Coskun, T. and Yavuz, C. (2004) A Case of a Diaphragmatic Rupture Complicated with Lacerations of Stomach and Spleen Caused by a Violent Cough Presenting with Mediastinal Shift. Annals of the Academy of Medicine, Singapore, 33, 649-650.

[4] Vinnicombe, Z., Little, M. and Wan, A. (2016) An Unusual Diaphragmatic Hernia with Gastric Perforation and Sub-Acute Presentation. The Annals of the Royal College of Surgeons of England, 98, e181-e383. https://doi.org/10.1308/rcsann.2016.0228

[5] Okyere, I., Okyere, P. and Komla Glover, P.S. (2019) Traumatic Right Diaphragmatic Rupture with Hepatothorax in Ghana: Two Rare Cases. The Pan African Medical Journal, 33, Article No. 256. https://doi.org/10.11604/pamj.2019.33.256.17061

[6] Yilmaz, M., Isik, B., Ara, C., Yilmaz, S., Kutlu, R., Kocak, Ö., et al. (2006) Gastric Perforation during Chest Tube Placement for Acute Diaphragmatic Rupture and Review of the Literature. Injury Extra, 37, 71-75. https://doi.org/10.1016/j.injury.2005.08.006

[7] Dwivedi, S., Banode, P., Gharde, P., Bhatt, M. and Johrapurkar, S.R. (2010) Treating Traumatic Injuries of the Diaphragm. Journal of Emergencies, Trauma, and Shock, 3, 173-176. https://doi.org/10.4103/0974-2700.62122

[8] Akar, E. and Kaya, H. (2017) Traumatic Rupture of the Diaphragm: A 22-Patient Experience. Biomedical Research, 28, 8640-8644.

[9] Simpson, J., Lobo, D.N., Shah, A.B. and Rowlands, B.J. (2000) Traumatic Diaphragmatic Rupture: Associated Injuries and Outcome. Annals of the Royal College of Surgeons of England, 82, 97-100.

[10] Wani, N., Kosar, T., Ahmad, A. and Yusuf, M. (2010) Traumatic Diaphragmatic Rupture with Delayed Gastric Incarceration. Journal of Emergencies Trauma, and Shock, 3, 306. http://www.onlinejets.org/text.asp?2010/3/3/306/66539 https://doi.org/10.4103/0974-2700.66539 